\title{
Design médical
}

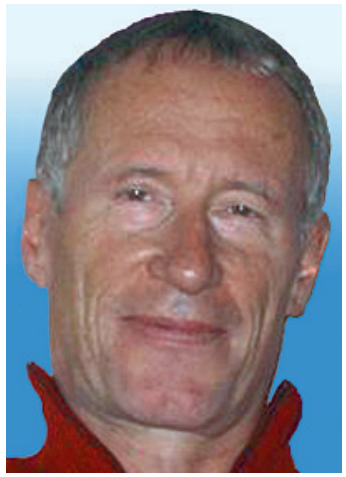

Erhard Taverna

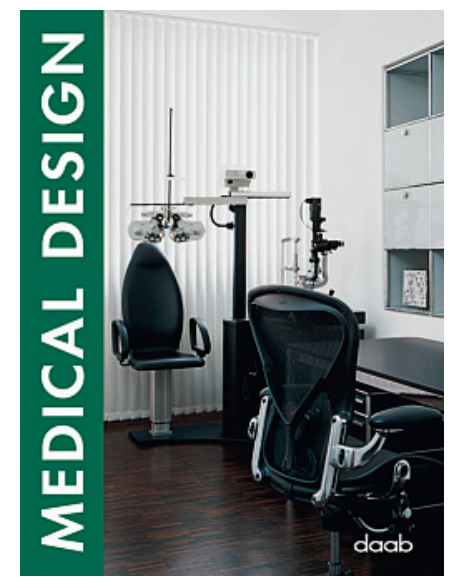

1 Medical Design.

Cologne: daab GmbH; 2006.

www.daab-online.com. 399 pages.

erhard.taverna@saez.ch
Durant les années passées en cabinet privé, les catalogues de meubles design n'ont cessé de me rappeler mes désirs quant à l'organisation de mon lieu de travail à mon arrivée. Mais l'agrandissement de l'entrepôt de tapis par celui qui avait été autrefois un petit marchand posa de sérieuses limites à mes rêves architecturaux. En outre, mon partenaire releva, fort à propos, les attentes simples de notre clientèle campagnarde à laquelle le nouvel arrivant devait s'adapter. Donc pas de cage d'escalier futuriste, mais de préférence une sonnerie faite de trois cloches de vache, pas de jeux de lumière ni de couleurs osées, pas de meubles élégants en matériaux raffinés ni d'objets véritablement design, mais des plafonds en bois et des sols en feutre gris anthracite résistant à la pression des bottes. Pas d'articles «Porsche design» sur le bureau, pas de photos d'îles paradisiaques aux murs, pas d'accessoires en cuir, aucun tape-à-l'œil, rien que du solide de production locale.

Parfois, il vaut la peine de se référer à l'histoire du design quand elle permet de capitonner les duretés existentielles de principes théoriques. Vitruve, architecte romain au service de Jules César, estimait qu'une structure est esthétique lorsqu'elle combine l'utile, la technique et le beau. Cette combinaison reste valable mais elle s'est enrichie aujourd'hui du symbole. A l'ère du tout marketing, les stylistes sont conscients du message transmis par les produits. Max Bill (19081994), célèbre architecte et designer suisse dont l'œuvre a été commentée dans tous les médias à l'occasion du $100^{\mathrm{e}}$ anniversaire de sa naissance, a, en sa qualité d'enseignant et d'artiste, fortement marqué notre compréhension du design. Les architectes modernes disent qu'un produit parle lorsque l'objet est à sa place, met en scène un message, fait le lien entre une esthétique formelle et un contenu raisonné. Les sièges monocoques en polyester, aluminium ou contre-plaqué transmettent un message différent de celui des chaises en gobelin avec accoudoirs rembourrés.

Les cabinets médicaux, salles d'attente, foyers d'hôpital et services d'admission trahissent, volontairement ou non, la personnalité des fournisseurs de prestations médicales concernés. Ils communiquent la confiance en soi et les compétences professionnelles, l'attention portée à autrui et la fiabilité. Actuellement, les exemples les plus extrêmes sont fournis par les décors d'une maternité; ici, les designers donnent libre cours à leur fantaisie pour créer une ambiance joyeuse et sécurisante. L'ouvrage «Medical Design», publié en Allemagne il y a un peu plus de deux ans, pré- sente les tendances actuelles au moyen de 60 projets du monde entier [1]. Il montre ce qu'est une bonne architecture d'hôpital, de cabinet médical, de pharmacie et autre institution de la santé quand elle s'inspire de l'architecture hôtelière, de ses matériaux et de ses couleurs. Des photos, plans et esquisses conduisent dans des bureaux de planification d'architectes renommés, de Memphis au Japon en passant par l'Europe et la Chine. Les illustrations sont accompagnées d'un commentaire succinct en anglais, allemand, espagnol, français et italien et de brèves coordonnées sur l'architecte, l'objet et l'année de construction. Les planificateurs allient de plus en plus souvent locaux de technique médicale et d'informatique, privés et publics, à des salles de séjour, cafés et hôtels. Ils optimisent le fonctionnement des processus de manière si naturelle que c'est finalement la qualité de l'organisation qui détermine le nombre de patients et donc le rendement économique de l'entreprise. Dans ce livre, on ne voit qu'une fois un feu brûler dans une cheminée et des bûches épaisses se trouver à portée de main près d'une chaise-longue tendue de rouge. La plupart des locaux reflètent une surface lisse monochrome, sont froidement fonctionnels, aérodynamiques, décoratifs et aseptiques. L'ombre de «Lost in Translation» plane sur la plupart des photos qui font penser à des halls d'embarquement, à des vaisseaux intergalactiques, à des musées d'art privés, à des saunas pour gens riches, à des laboratoires high-tech et à des paquebots de luxe. Mais on n'y voit aucune âme qui vive. Il y faudrait des personnes grandes et sveltes, sorte de mannequins vêtus de t-shirts dans le pur style Armani ou arborant le look Prada en peluche, accompagnées de thérapeutes vigoureux et portant le survêtement stylisé de la clinique, avec son logo discret mais bien visible.

Devant ma fenêtre, les abeilles bourdonnent autour des abricotiers en fleur. Mon regard englobe la vallée où le printemps joue, en parfait designer, avec la palette des couleurs pour me donner une vue imprenable. De nombreux patients viennent en consultation directement depuis leur lieu de travail, sentent le fumier, le foin, l'huile de machine, la cuisine, le bois ou la peinture. Ils portent des vestes à carreaux, rarement des habits à la mode. Ils sont gros ou minces, ont des visages connus ou inconnus, sont dépressifs ou heureux de vivre, courageux, mélancoliques, taciturnes ou communicatifs, sont d'ici ou d'ailleurs. Qu'est-ce que je veux de plus?

Erhard Taverna 